\title{
Exact asymptotically flat charged hairy black holes with a dilaton potential
}

\author{
${ }^{(1)}$ Andrés Anabalón, ${ }^{(2)}$ Dumitru Astefanesei and ${ }^{(3,4)}$ Robert Mann. \\ (1) Departamento de Ciencias, Facultad de Artes Liberales y \\ Facultad de Ingeniería y Ciencias, Universidad Adolfo Ibáñez, Viña del Mar, Chile. \\ ${ }^{(2)}$ Instituto de Física, Pontificia Universidad Católica de Valparaíso, \\ Casilla 4059, Valparaíso, Chile. \\ (3) Department of Physics and Astronomy, University of Waterloo, \\ Waterloo, Ontario, Canada N2L 3 G1. \\ (4) Perimeter Institute, 31 Caroline Street North Waterloo, Ontario Canada N2L 2 Y5.
}

November 6, 2013

\begin{abstract}
We find broad classes of exact 4-dimensional asymptotically flat black hole solutions in EinsteinMaxwell theories with a non-minimally coupled dilaton and its non-trivial potential. We consider a few interesting limits, in particular, a regular generalization of the dilatonic Reissner-Nordström solution and, also, smooth deformations of supersymmetric black holes. Further examples are provided for more general dilaton potentials. We discuss the thermodynamical properties and show that the first law is satisfied. In the non-extremal case the entropy depends, as expected, on the asymptotic value of the dilaton. In the extremal limit, the entropy is determined purely in terms of charges and is independent of the asymptotic value of the dilaton. The attractor mechanism can be used as a criterion for the existence of the regular solutions. Since there is a 'competition' between the effective potential and dilaton potential, we also obtain regular extremal black hole solutions with just one $\mathrm{U}(1)$ gauge field turned on.
\end{abstract}

\section{Introduction.}

No-hair theorems [1] apply to stationary asymptotically flat black holes in theories of gravity coupled to scalar fields with an interaction potential that is convex [2] or positive semidefinite [3]. It is perhaps unsurprising that if one or more of these assumptions are relaxed, then the theorem does not hold. As is well known, the scalar potential of compactified supergravity 1 (see, 44 for a discussion in the context of no-hair theorems) does not necessary satisfy the constraints mentioned above and one can ask if the no-hair theorems could be evaded for some potentials of scalar fields. It is by now well known that, in asymptotically anti de Sitter (AdS) spacetimes, there exist indeed (numerical, as well as exact) scalar-hairy black holes for some scalar field potentials (see, e.g., [5, 6, 7]).

In AdS, the existence of hairy black holes is related to the fact that the asymptotic value of the scalar field potential can be reabsorbed in an effective cosmological constant that differs from the true cosmological constant of the theory. Interestingly, it has been suggested in [6] that the effective cosmological constant could vanish if the parameters of the scalar field potential are properly adjusted and numerical evidence for the existence of the hairy asymptotically flat black holes was presented.

\footnotetext{
${ }^{1}$ The effective potential typically has negative regions but supersymmetry ensures the total energy is always positive.
} 
Therefore, by fine tuning the cosmological constant or the scalar field potential so that the effective cosmological vanishes, one can obtain scalar field hair for asymptotically flat black holes. The scalar field potentials considered in [6] are not positive semidefinite and the weak energy condition is violated.

Behind the no-hair theorems there is a simple physical picture: the matter fields left in the exterior after the gravitational collapse would eventually be 'swallowed' by the black hole itself or be radiated away to infinity 2 Since the 'hair' is located outside the horizon, one could ask how is it possible that matter can hover in a strong gravitational field without collapsing completely? Intuitively, the answer could be that this is possible if the internal pressure is sufficiently large. However, this kind of intuition works just near the horizon and it should be clear that the the non-linear (self-interacting) character of the matter fields is, in fact, at the basis of the existence of hairy black holes. A nice heuristic picture was presented in [8]: the self-interaction of matter fields (together with the gravitational interaction) is responsible for the fact that the near-horizon hair does not collapse into the black hole while the far-region hair does not escape to infinity. Therefore, the hair should extend some way out from the event horizon so that the degrees of freedom near the horizon are bound together with the ones that tend to be radiated away at infinity.

In this paper, we present exact non-extremal asymptotically flat charged hairy black holes with a dilaton potential and investigate their thermodynamic properties 3 In particular, we are able to recover some of the solutions of [11] when the dilaton potential vanishes. In [11], the authors found the black hole solutions by rewriting the equations of motion as Toda equations. However, when there is a non-trivial dilaton potential, this method of solving the equations of motion is not very useful. Instead, we use the metric ansatz of [7] to obtain charged hairy black hole solutions, which are asymptotically flat.

In the non-extremal case, the near horizon data depend on the value of the dilaton at the horizon. In the extremal case, due to the attractor mechanism [12, the near horizon data are completely fixed by the electric and magnetic charges and so the attractor mechanism acts as a no-hair theorem [1] for the extremal black holes [13. The attractor mechanism is a useful tool in investigating the existence of the regular extremal black hole solutions.

The remainder of the paper is organized as follows: In Section 2, we construct the hairy black hole solutions in flat space. First, we obtain a general solution and then we discuss some concrete examples which are related to some known solutions when the dilaton potential vanishes. In Section 3 we explicitly compute the thermodynamic quantities and show that the first law is satisfied. In Section 4 we use the attractor equations to investigate the extremal limit. We show that at zero temperature, contrary to the case for which the dilaton potential vanishes, there exist regular solutions with only one gauge field (electric or magnetic) turned on. Finally, we end with a detailed discussion of our results in Section 5 including the energy conditions. In the appendices we present more general solutions and detail our progress in looking for analytic solutions.

\footnotetext{
${ }^{2}$ The limited information about the matter that collapsed is reflected only in the number of conserved charges that are conserved surface integrals in the asymptotically flat region. 'Hair' does not refer to those fields, which are associated with conserved charges.

${ }^{3}$ Exact charged hairy black holes in Anti-de Sitter spacetime will be presented elsewhere 9 . Exact hairy black hole solutions in Anti-de Sitter when the gauge fields are turned off were presented in [7] and their holographic properties were investigated in [10, 7].
} 


\section{Non-extremal solutions.}

In this section, we construct static asymptotically flat non-extremal black holes for a model with one scalar field (dilaton) non-minimally coupled to a gauge field and a non-trivial dilaton potential. For an exponential coupling, the equations of motion can be solved exactly with a special metric ansatz and the dilaton potential can be explicitly obtained. Interestingly enough, in this way we obtain analytic generalizations of some well known solutions, in particular the dilatonic Reissner-Nordström and other solutions for which the extremal limits are supersymmetric. The action we are interested in is

$$
I\left[g_{\mu \nu}, A_{\mu}, \phi\right]=\frac{1}{2 \kappa} \int d^{4} x \sqrt{-g}\left[R-\frac{1}{4} e^{\gamma \phi} F^{2}-\frac{1}{2} \partial_{\mu} \phi \partial^{\mu} \phi-V(\phi)\right]
$$

where the gauge coupling and potential are functions of the dilaton and we use the convention $\kappa=$ $8 \pi G_{N}$. Since we set $c=1=\hbar,[\kappa]=M_{P}^{-2}$ where $M_{P}$ is the reduced Planck mass. The equations of motion for the gauge field, dilaton, and metric are

$$
\begin{gathered}
\nabla_{\mu}\left(e^{\gamma \phi} F^{\mu \nu}\right)=0 \\
\frac{1}{\sqrt{-g}} \partial_{\mu}\left(\sqrt{-g} g^{\mu \nu} \partial_{\nu} \phi\right)-\frac{\partial V}{\partial \phi}-\frac{1}{4} \gamma e^{\gamma \phi} F^{2}=0 \\
R_{\mu \nu}-\frac{1}{2} g_{\mu \nu} R=\frac{1}{2}\left[T_{\mu \nu}^{\phi}+T_{\mu \nu}^{E M}\right]
\end{gathered}
$$

where the stress tensors of the matter fields are

$$
T_{\mu \nu}^{\phi}=\partial_{\mu} \phi \partial_{\nu} \phi-g_{\mu \nu}\left[\frac{1}{2}(\partial \phi)^{2}+V(\phi)\right] \quad, \quad T_{\mu \nu}^{E M}=e^{\gamma \phi}\left(F_{\mu \alpha} F_{\nu}^{\cdot \alpha}-\frac{1}{4} g_{\mu \nu} F^{2}\right)
$$

It is still very difficult to find exact solutions to the system of coupled differential equations above when the dilaton potential does not vanish, even for simple potentials [14, 15, 16]. For simplifying our analysis, we use the following spherically symmetric ansatz for the metric:

$$
d s^{2}=\Omega(x)\left[-f(x) d t^{2}+\frac{\eta^{2} d x^{2}}{f(x)}+d \theta^{2}+\sin ^{2} \theta d \varphi^{2}\right]
$$

where the parameter $\eta$ was introduced to obtain a dimensionless radial coordinate $x$ and $\Omega(x)$ is the conformal factor. Written in this way, it is clear that $\eta$ is going to be the only constant of integration of gravitational origin in the solution (it does not appear in the action) and so it should be related to the mass of the solution. This is the most general static asymptotically flat solution and so it is characterized by only two unknown functions.

The equation of motion for the gauge field and Bianchi identity can be solved via the following ansatz for the field strength:

$$
F=Q e^{-\gamma \phi} d x \wedge d t+P \sin \theta d \varphi \wedge d \theta
$$

Using the observation that the only terms involving the dilaton potential in the Einstein equations are of the form $\delta_{\nu}^{\mu} V(\phi)$, we can use combinations for which the dilaton potential cancels out. We then explicitly obtain a differential equation that involves only the dilaton and conformal factor $\Omega(x)$ :

$$
E_{t}^{t}-E_{x}^{x}=0 \rightarrow \phi^{\prime 2}=\frac{-2 \Omega^{\prime \prime} \Omega+3 \Omega^{\prime 2}}{\Omega^{2}}
$$

where $\Omega^{\prime}$ is the derivative with respect to the coordinate $x$ and $E_{\mu}^{\nu}$ are the Einstein field equations. 
For some special conformal factors $\Omega(x)$, the equation above can be exactly solved and, in this way, we are able to obtain the analytic solutions presented below. We would also like to point out that one can get the 'hairy' Reissner-Nordström solution by taking the limit $\gamma=0$.

The strategy for finding solutions is to use the same conformal factor as in [7]

$$
\Omega(x)=\frac{\nu^{2} x^{\nu-1}}{\eta^{2}\left(x^{\nu}-1\right)^{2}}
$$

then find the scalar field from (8). We would like to emphasize that the new parameter $\nu$ labels different solutions, for example $\nu=1$ and $\gamma=0$ corresponds to Reissner-Nordström black hole, and for $\nu>1$ we obtain 'hairy' solutions.

From (8) it follows immediately that

$$
\phi(x)=l_{\nu}^{-1} \ln (x)+\phi_{0}
$$

where $l_{\nu}=\left(\nu^{2}-1\right)^{-\frac{1}{2}}$ plays the role of a characteristic length scale of the dilaton. There is a \pm ambiguity in the integration of (8), which corresponds to a discrete degeneration in the black hole family. Indeed, from (9) it follows that the conformal factor has a pole of order two at $x=1$ where the conformal infinity is 'located'. The fact that (9) is regular in the region $x \in(0,1)$ and $x \in(1, \infty)$ allows to pick any of these intervals as the domain of coordinates, one corresponding to a negative scalar and the other corresponding to a positive one.

The remaining metric function satisfies the following differential equation (we also have $E_{\theta}^{\theta}=E_{\phi}^{\phi}$ ):

$$
E_{t}^{t}-E_{\theta}^{\theta}=0 \rightarrow \frac{d}{d x}\left(\Omega(x) f^{\prime}(x)\right)+2 \eta^{2} \Omega(x)-e^{-\gamma \phi} Q^{2}-e^{\gamma \phi} \eta^{2} P^{2}=0
$$

which can be exactly integrated to yield

$$
\begin{aligned}
f(x) & =f_{0}+\frac{\eta^{2}}{\nu}\left(x^{2}+\frac{2 x^{2-\nu}}{\nu-2}\right)+f_{1}\left(\frac{x^{\nu+2}}{\nu+2}-x^{2}+\frac{x^{2-\nu}}{2-\nu}\right) \\
& +\frac{Q^{2} \eta^{2}}{(1-p) \nu^{2}}\left(\frac{x^{3-p+\nu}}{3-p+\nu}+\frac{x^{3-p-\nu}}{3-p-\nu}-2 \frac{x^{3-p}}{3-p}\right)+\frac{P^{2} \eta^{4}}{(1+p) \nu^{2}}\left(\frac{x^{3+p+\nu}}{3+p+\nu}+\frac{x^{3+p-\nu}}{3+p-\nu}-2 \frac{x^{3+p}}{3+p}\right)
\end{aligned}
$$

where $f_{0}$ and $f_{1}$ are constants of integration and $p=\gamma / l_{\nu}=\gamma \sqrt{\nu^{2}-1}$.

From now on, we shall consider only asymptotically flat solutions. To enforce this condition, we require

$$
\lim _{x \rightarrow 1} \Omega(x) f(x)=1
$$

and, consequently, we fix $f_{0}$ to obtain

$$
\begin{aligned}
f(x) & =\frac{\eta^{2}}{\nu}\left(x^{2}+\frac{2 x^{2-\nu}}{\nu-2}-\frac{\nu}{\nu-2}\right)+f_{1}\left(\frac{x^{\nu+2}}{\nu+2}-x^{2}+\frac{x^{2-\nu}}{2-\nu}+\frac{\nu^{2}}{\nu^{2}-4}\right) \\
& +\frac{Q^{2} \eta^{2}}{(1-p) \nu^{2}}\left(\frac{x^{3-p+\nu}}{3-p+\nu}+\frac{x^{3-p-\nu}}{3-p-\nu}-2 \frac{x^{3-p}}{3-p}-\frac{2 \nu^{2}}{(3-p)(3-p+\nu)(3-p-\nu)}\right) \\
& +\frac{P^{2} \eta^{4}}{(1+p) \nu^{2}}\left(\frac{x^{3+p+\nu}}{3+p+\nu}+\frac{x^{3+p-\nu}}{3+p-\nu}-2 \frac{x^{3+p}}{3+p}-\frac{2 \nu^{2}}{(3+p)(3+p+\nu)(3+p-\nu)}\right)
\end{aligned}
$$

Let us elaborate a bit more on the boundary condition (13). In canonical areal coordinates, the transverse section of the black hole should be multiplied by $r^{2}$ at infinity. It follows from (6) that there is a change of coordinates such that

$$
\Omega=r^{2}+\ldots
$$


where the dots stand for subleading terms (a more precise definition is given below). This change of coordinates is compatible with the asymptotic form of Minkowski spacetime if the lapse function goes as

$$
\Omega f=1+\ldots
$$

which uniquely fixes (13).

A detailed analysis of (14) shows that it has regular limits when $\nu= \pm 2, p= \pm 3, p=\nu \pm 3$, $p=-\nu \pm 3$ and it is singular at $p= \pm 1$. The singularities can be cured by picking

$$
f_{1}=\frac{\eta^{2} Q^{2}}{(p-1) \nu^{2}}-\frac{\eta^{4} P^{2}}{(p+1) \nu^{2}}+\alpha+\frac{\eta^{2}(\nu+2)}{\nu^{2}}
$$

it is easy to see that the function $f(x)$ has smooth limits for all the values of the parameters.

This general solution is complicated, but we are going present the results for some concrete examples corresponding to specific values of $\gamma$ only. In particular, we are going to consider the $\gamma=\sqrt{3}$ case for which there indeed exists a smooth limit despite the divergence in the denominator of (14). In the extremal case, at the horizon the metric function should satisfy $f^{\prime \prime}\left(x_{h}\right)=$ constant and $f^{\prime}\left(x_{h}\right)=0$.

Once we obtain the metric, it is straightforward to algebraically find the potential so that the field equations are satisfied. In general, the potential is a function of six parameters: besides $\gamma, \nu, Q$, and $P$ it is possible to introduce a cosmological constant and an extra parameter, $\alpha$, which appears as an overall multiplication constant in the potential, after a redefinition of $f_{1}$.

A general class of solutions with the gauge fields turned on and with cosmological constant is going to be presented in [9]. In what follows, we focus on asymptotically flat solutions when the gauge fields are turned on and there is a non-trivial dilaton potential - for these solutions the dilaton potential is vanishing at the boundary. With the ansatz ([6), it is possible to construct a five parameter family of dyonic black hole solutions.

Dyonic black holes for the Einstein-Maxwell-Dilaton theory that can be embedded in string theory are known for $\gamma=1$ and $\gamma=\sqrt{3}$. In what follows, we present the extension of the $\gamma=1$ solution. For

$\gamma=\sqrt{3}$, we have also found a dyonic black hole. However, in this case, the potential has a part that is proportional with the magnetic charge and the only way to consider the limit of vanishing potential is when the magnetic charge is also vanishing. In this limit, the solution matches the electrically charged Kaluza-Klein black hole. We are going to also present a generalization of 'hairy' Reissner-Nordström black holes for which the scalar field and gauge field are not coupled, but the dilaton potential is non-trivial.

More general solutions are listed in Appendices $\mathrm{A}$ and $\mathrm{B}$ but, for simplicity, in this section we are going to focus only on some deformations of solutions that can be embedded in supergravity.

\subsection{Solutions with a non-trivial dilaton potential}

\subsection{1 $\gamma=1$}

To obtain this solution we should also consider the limit $\nu \rightarrow \infty-$ a more general family of solutions with arbitrary $\gamma$ is presented in Appendix A.

To obtain the limit $\nu \rightarrow \infty$ in a smooth way, we change the coordinates as

$$
x \rightarrow x^{\frac{1}{\nu}}, \quad t \rightarrow \frac{t}{\nu}
$$

and rescale the 2 -sphere as

$$
d \theta^{2}+\sin ^{2} \theta d \varphi^{2} \rightarrow \nu^{-2}\left(d \theta^{2}+\sin ^{2} \theta d \varphi^{2}\right),
$$


in which case the $\nu^{2}$ of the conformal factor (9) cancels out.

After taking the limit, we obtain

$$
\begin{gathered}
\Omega(x)=\frac{x}{\eta^{2}(x-1)^{2}} \quad, \quad \phi(x)=\ln (x) \\
d s^{2}=\Omega(x)\left[-f(x) d t^{2}+\frac{\eta^{2} d x^{2}}{x^{2} f(x)}+d \theta^{2}+\sin ^{2} \theta d \varphi^{2}\right]
\end{gathered}
$$

We emphasize that the $g_{x x}$ metric component is now different than the one for finite $\nu$ (see (6) ). One can work with an arbitrary parameter $\gamma$ in the dilatonic coupling. The field equations solvable and the solution is presented in Appendix A. However, in this section, we describe only the $\gamma=1$ case, which is very simple and can be smoothly connected with a solution of $\mathcal{N}=4$ supergravity. To completely characterize the solution, apart from the conformal factor and dilaton (20), we have the following remaining data:

$$
\begin{gathered}
A=\frac{Q}{x} d t+P \cos \theta d \varphi \\
V(\phi)=\alpha[2 \phi+\phi \cosh (\phi)-3 \sinh (\phi)] \\
f(x)=\frac{\eta^{2}(x-1)^{2}}{x}+\left[\frac{x}{4}-\frac{1}{4 x}-\frac{1}{2} \ln (x)\right] \alpha+\frac{\eta^{2}(x-1)^{3}}{2 x}\left(\eta^{2} P^{2}-x^{-1} Q^{2}\right)
\end{gathered}
$$

One can easily see that at the boundary, $x=1$, the dilaton is vanishing and so does the dilaton potential. The limit for vanishing dilaton potential is obtained when $\alpha=0$.

Let us now show explicitly that the metric is asymptotically flat by studying the boundary $x=1$. To do this we look for a change of coordinates such that at infinity the sphere is multiplied by $r^{2}$

$$
\Omega(x)=r^{2}+O\left(r^{-4}\right),
$$

which is given for, the $x<1$ black holes, by

$$
x=1-\frac{1}{\eta r}+\frac{1}{2 \eta^{2} r^{2}}-\frac{1}{8 \eta^{3} r^{3}}+\frac{1}{2^{7} \eta^{5} r^{5}}
$$

Now, it is possible to see that the metric is asymptotically flat

$$
\begin{gathered}
g_{t t}=\Omega(x) f(x)=1-\frac{\alpha+6 \eta^{2}\left(\eta^{2} P^{2}-Q^{2}\right)}{12 \eta^{3} r}+O\left(r^{-2}\right) \\
g_{r r}^{-1}=\frac{x^{2} f(x)}{\eta^{2} \Omega(x)}\left(\frac{d r}{d x}\right)^{2}=1-\frac{\alpha+6 \eta^{2}\left(\eta^{2} P^{2}-Q^{2}\right)}{12 \eta^{3} r}+O\left(r^{-2}\right) .
\end{gathered}
$$

The same change of coordinates exists for $x>1$. The scalar field potential is everywhere regular, except at the spacetime singularities.

\subsection{2 $\gamma=\sqrt{3}$}

Let us consider now another interesting solution, which can be smoothly connected with the electrically charged Kaluza-Klein black hole when the dilaton potential is vanishing. This case corresponds to

$$
\gamma=\left(\frac{\nu+1}{\nu-1}\right)^{1 / 2}
$$


when $\nu=2$ - once again, we present a more general family of solutions with $\gamma$ arbitrary in Appendix B. The metric is

$$
d s^{2}=\Omega(x)\left[-f(x) d t^{2}+\frac{\eta^{2} d x^{2}}{f(x)}+d \theta^{2}+\sin ^{2} \theta d \varphi^{2}\right]
$$

where the metric function, $f(x)$, and conformal factor, $\Omega(x)$, are

$$
\begin{gathered}
f(x)=\alpha\left[2 \ln (x)+\frac{x^{4}}{2}+\frac{3}{2}-2 x^{2}\right]+\frac{\eta^{4} P^{2}}{384}\left(x^{2}-1\right)^{3}\left(3 x^{2}+1\right)-\frac{\eta^{2}\left(x^{2}-1\right)^{3} Q^{2}}{16 x^{2}}+\frac{\eta^{2}\left(x^{2}-1\right)^{2}}{4} \\
\Omega(x)=\frac{4 x}{\eta^{2}\left(x^{2}-1\right)^{2}}
\end{gathered}
$$

The dilaton and gauge potential are

$$
\phi=\sqrt{3} \ln (x) \quad, \quad A=-\frac{Q}{2 x^{2}} d t+P \cos \theta d \varphi
$$

and the dilaton potential is

$$
V(\phi)=\alpha\left[\sinh (\sqrt{3} \phi)+9 \sinh \left(\frac{\phi}{\sqrt{3}}\right)-\frac{12 \phi}{\sqrt{3}} \cosh \left(\frac{\phi}{\sqrt{3}}\right)\right]-\frac{e^{-\frac{\phi}{\sqrt{3}}} \eta^{4} P^{2}\left(e^{\frac{2 \phi}{\sqrt{3}}}-1\right)^{5}}{2^{7}}
$$

Again we observe that at the boundary $x=1$ the dilaton is vanishing, and the dilaton potential likewise vanishes there. However, the limit for which the dilaton potential is vanishing corresponds this time to $\alpha=0$ and $P=0$ - in this limit we recover the electrically charged Kaluza-Klein black hole. One important observation is that, unlike the extremal limit of an electrically charged KaluzaKlein black hole that is singular, our solution has a regular extremal limit due to the presence of the dilaton potential. We are going to discuss this point in more detail in Section 4.

\subsubsection{Hairy Reissner-Nordström black hole}

We consider the theory for which gauge coupling does not depend of the dilaton, namely $\gamma=0$. As follows from the equations of motion, this is a consistent truncation of the non-minimally coupled theory. Therefore, following the results of Section 2, the scalar field and the conformal factor are given by (10) and (9), respectively. The integration of the field equation for $f(x)$, is given by the expression (14) when $\gamma=p=0$ and yields

$$
\begin{gathered}
f(x)=\frac{\eta^{2}}{\nu}\left(x^{2}+\frac{2 x^{2-\nu}}{\nu-2}-\frac{\nu}{\nu-2}\right)+f_{1}\left(\frac{x^{\nu+2}}{\nu+2}-x^{2}+\frac{x^{2-\nu}}{2-\nu}+\frac{\nu^{2}}{\nu^{2}-4}\right) \\
+\frac{\left(Q^{2} \eta^{2}+P^{2} \eta^{4}\right)}{\nu^{2}}\left(\frac{x^{3+\nu}}{3+\nu}+\frac{x^{3-\nu}}{3-\nu}-2 \frac{x^{3}}{3}-\frac{2 \nu^{2}}{3(3+\nu)(3-\nu)}\right)
\end{gathered}
$$

which upon using the definitions $q=\frac{2 \eta^{2}\left(Q^{2}+\eta^{2} P^{2}\right)}{3}, f_{1}=\frac{\alpha}{\nu^{2}}+\frac{\eta^{2}(\nu+2)}{\nu^{2}}$ yields

$$
\begin{aligned}
f(x) & =\frac{x^{2-\nu} \eta^{2}}{\nu^{2}}\left(x^{\nu}-1\right)^{2}+\alpha\left[\frac{1}{\nu^{2}-4}-\left(1+\frac{x^{-\nu}}{\nu-2}-\frac{x^{\nu}}{\nu+2}\right) \frac{x^{2}}{\nu^{2}}\right] \\
& +q\left[\frac{1}{\left(\nu^{2}-9\right)}-\left(1+\frac{3}{2} \frac{x^{-\nu}}{\nu-3}-\frac{3}{2} \frac{x^{\nu}}{3+\nu}\right) \frac{x^{3}}{\nu^{2}}\right]
\end{aligned}
$$


Unlike the previous case, the dilaton potential has a part that depends on both electric and

magnetic charges, through the combination $q=\frac{2 \eta^{2}\left(Q^{2}+\eta^{2} P^{2}\right)}{3}$, which appears also in the metric. The dilaton potential is more complicated:

$$
\begin{aligned}
V(\phi) & =\frac{2 \alpha}{\nu^{2}}\left(\frac{\nu-1}{\nu+2} \sinh \left((1+\nu) \phi l_{\nu}\right)+\frac{\nu+1}{\nu-2} \sinh \left((1-\nu) \phi l_{\nu}\right)+4 \frac{\nu^{2}-1}{\nu^{2}-4} \sinh \left(\phi l_{\nu}\right)\right) \\
& +\frac{3 q e^{2 \phi l_{\nu}}}{4 \nu^{4}}\left(\frac{\nu-1}{\nu+3} e^{2 \nu \phi l_{\nu}}+\frac{\nu+1}{\nu-3} e^{-2 \nu \phi l_{\nu}}\right) \\
& +\frac{q\left(\nu^{2}-1\right) e^{2 \phi l_{\nu}}}{\nu^{4}}\left(\frac{\nu-3}{\nu+3} e^{\nu \phi l_{\nu}}+\frac{\nu+3}{\nu-3} e^{-\nu \phi l_{\nu}}+\frac{1}{2} \frac{\left(8 \nu^{2}-27\right)}{\left(\nu^{2}-9\right)}\right) \\
& -\frac{q\left(\nu^{2}-4\right) e^{-\phi l_{\nu}}}{\nu^{2}\left(\nu^{2}-9\right)}\left(\frac{\nu-1}{\nu+2} e^{-\nu \phi l_{\nu}}+\frac{\nu+1}{\nu-2} e^{\nu \phi l_{\nu}}-4 \frac{\left(\nu^{2}-1\right)}{\left(\nu^{2}-4\right)}\right),
\end{aligned}
$$

and it has been arranged such that it is explicitly invariant under $\nu \rightarrow-\nu$. The gauge field is given by (7) with $\gamma=0$.

Let us close this section with a couple of comments. We observe, as expected, that a consistent limit for which the Reissner-Nordström solution is recovered is $\nu=1$ (which also means $\phi=0$ ) when the dilaton potential is vanishing. The potential vanishes when $\alpha=0$ and both charges also vanish (because we should have $q=0$ so that the part of the potential proportional to $q$ is also vanishing). In other words, there are no regular hairy Reissner-Nordström black holes with a vanishing dilaton potential, as expected from no-hair theorems.

\subsection{Solutions with a vanishing dilaton potential}

The solutions we found are regular and generalise some of the solutions that were previously known for the case of vanishing scalar potential. The solutions presented in [11, 17, 18, 19] were obtained by rewriting the equations of motion as Toda equations [11. This method is useful for obtaining solutions in certain cases with exponential couplings. However, it is not powerful enough to construct more general solutions with non-trivial moduli potentials.

We are interested in enough simple solutions (with regular supersymmetric limits), but a whole new family of solutions can be generated from our general solutions presented in Appendices.

We are going to explicitly show how an old known solution can be recovered from the new solutions with a non-trivial potential that we have constructed. This solution was presented in [20] (see, also, [19] for a detailed discussion of its thermodynamical properties and the extremal limit in the context of attractor mechanism) and has the following form:

$$
\begin{gathered}
d s^{2}=-a(r)^{2} d t^{2}+a(r)^{-2} d r^{2}+b(r)^{2} d \Omega^{2} \\
\exp (2 \phi)=\frac{r+\Sigma}{r-\Sigma}, a^{2}=\frac{\left(r-r_{+}\right)\left(r-r_{-}\right)}{r^{2}-\Sigma^{2}}, b^{2}=r^{2}-\Sigma^{2}
\end{gathered}
$$

with

$$
r_{ \pm}=M \pm \sqrt{M^{2}+\Sigma^{2}-Q^{2}-P^{2}}
$$

We emphasize that the scalar charge $\Sigma$ is not an independent parameter:

$$
\Sigma=\frac{P^{2}-Q^{2}}{2 M}
$$


The scalar charge plays a role in the first law when the asymptotic value of the dilaton does vary [21. The extremal limit of the above solution is obtained by letting $r_{+}=r_{-}$and the corresponding solution can be embedded in $\mathcal{N}=4$ supergravity.

We can reobtain this solution from our solution $\gamma=1$ when the dilaton potential is vanishing $(\alpha=0)$. To find the right change of coordinates, first we match the scalars and we get:

$$
x=\frac{r+\Sigma}{r-\Sigma} \Longrightarrow \phi=\ln \left(\frac{r+\Sigma}{r-\Sigma}\right)
$$

Then, to obtain the same expressions for the gauge field, we redefine $Q=-\frac{q}{2 \Sigma}$ in (22) and so we get the following gauge potential:

$$
A=-\frac{q}{2 \Sigma} \frac{r-\Sigma}{r+\Sigma} d t+P \cos \theta d \varphi
$$

The last step is to fix $\eta$ by matching the $b^{2}$ of (38) with the conformal factor $\Omega$ :

$$
\Omega(x(r))=r^{2}-\Sigma^{2}, \quad \eta=\frac{1}{2 \Sigma}
$$

Once we have the right change of coordinates

$$
d x=-\frac{2 \Sigma d r}{(r-\Sigma)^{2}}, \quad \frac{\eta^{2} d x^{2}}{x^{2}}=\frac{d r^{2}}{\left(r^{2}-\Sigma^{2}\right)^{2}}
$$

it is straightforward to obtain

$$
a(r)=\frac{4 \Sigma r^{2}+\left(P^{2}-q^{2}\right) r+\Sigma q^{2}+\Sigma P^{2}-4 \Sigma^{3}}{4 \Sigma\left(r^{2}-\Sigma^{2}\right)}
$$

which matches (39) when the charges are rescaled with a factor of $1 / 2$ as it should be for a 2 -form field strength.

One can similarly show that the $\gamma=\sqrt{3}$ is smoothly connected with the electrically charged Kaluza-Klein black hole (that can be obtained by Kaluza-Klein reduction of the $5 d$ Schwarzschild black hole) of [17, 18].

\section{Hair and thermodynamical properties.}

To study the first law of thermodynamics for our black holes, we first compute the entropy and the temperature. In the coordinates we use to write our metric ansatz (6), the boundary is at $x=1$ and the horizon is at $x=x_{h}$. The temperature (an intensive quantity) is conformally invariant and so depends only on $f(x)$ and not on the conformal factor:

$$
T=\left.\frac{f^{\prime}(x)}{4 \pi \eta}\right|_{x=x_{h}}
$$

The entropy and mass can be also easily computed and we get

$$
S=\frac{\pi \Omega\left(x_{h}\right)}{G_{N}}, \quad M=\left.\frac{[\Omega(x) f(x)]^{\prime}}{2 \eta G_{N}}\right|_{x=1}
$$


To gain some intuition, let us first discuss the Schwarzschild solution ( $\nu=-1$ in Appendix B) in the coordinates we use

$$
\Omega=\frac{1}{\eta^{2}(x-1)^{2}} \quad, \quad f(x)=(x-1)^{2}\left[\eta^{2}+(x-1)\left(\eta^{2}+\frac{\alpha}{12}\right)\right]
$$

for which $x_{h}=\frac{\alpha}{\alpha+12 \eta^{2}}$. The change of coordinates to put the metric in the canonical Schwarzschild form is

$$
x=1-\frac{1}{\eta r}
$$

Using the general formulas for the temperature, entropy, and mass presented above, we obtain

$$
\begin{gathered}
M=\frac{1}{2 \eta G_{N}}\left(1+\frac{\alpha}{12 \eta^{2}}\right), \quad S=\frac{\pi}{G_{N}} \frac{1}{\eta^{2}\left(x_{h}-1\right)^{2}}=\frac{\pi}{G_{N}} \frac{1}{\eta^{2}}\left(1+\frac{\alpha}{12 \eta^{2}}\right)^{2} \\
T=\frac{\left(x_{h}-1\right)\left[8 \eta^{2}+\left(x_{h}-1\right)\left(\alpha+12 \eta^{2}\right)\right]}{16 \pi \eta}=\frac{\eta}{4 \pi}\left(1+\frac{\alpha}{12 \eta^{2}}\right)^{-1}
\end{gathered}
$$

from which we recover the well known results

$$
M=\frac{m}{G_{N}} \rightarrow S=\frac{4 \pi m^{2}}{G_{N}}, T=\frac{1}{8 \pi m}
$$

in the limit $\alpha \rightarrow 0$.

The only subtlety related to the first law in the coordinates we use comes from the fact that the horizon radius is dimensionless. However, the computations above can be straightforwardly generalized to our solutions. For concreteness, let us now discuss the $\gamma=\sqrt{3}$ solution. Similar but more involved computations can be done for the other solutions we have presented in the previous section.

With our conventions, the electric charge can be written as

$$
q=-\frac{Q}{4 \eta G_{N}}
$$

and the conjugate potential is $\Phi_{q}=\frac{Q}{2}\left(1-\frac{1}{x_{h}^{2}}\right)$ (which is, up to an additive constant, just the usual gauge potential $\left.\Phi_{q}=Q / x_{h}^{2}\right)$ in a gauge such that it vanishes at the boundary $x=1$ and it is finite at the horizon.

The expressions for the mass, temperature, and entropy for the $\gamma=\sqrt{3}$ solution are

$$
\begin{gathered}
M=\frac{32 \alpha+12 \eta^{2}-6 \eta^{2} Q^{2}+\eta^{4} P^{2}}{24 \eta^{3} G_{N}} \\
T=\frac{\left(x_{h}^{2}-1\right)^{2}}{4 \pi \eta}\left[\frac{2 \alpha}{x_{h}}+\frac{\eta^{4} x_{h}^{3} P^{2}}{16}-\frac{\eta^{2}\left(2 x_{h}^{2}+1\right) Q^{2}}{8 x_{h}^{3}}+\frac{\eta^{2} x_{h}}{x_{h}^{2}-1}\right] \\
S=\frac{4 \pi x_{h}}{\eta^{2}\left(x_{h}^{2}-1\right)^{2} G_{N}}
\end{gathered}
$$

For simplicity, one can consider the case $P=0$. Since the radius of the horizon, $x_{h}$, cannot be explicitly obtained from $f\left(x_{h}\right)=0$, one can verify the first law by using the chain rule to get the variations with respect to the independent parameters $Q$ and $\eta$ :

$$
d M(q, \eta)=T d S(q, \eta)+\Phi_{q} d q
$$




\section{Extremal limit and attractor mechanism.}

The role of attractor mechanisms [12] for computing the entropy of extremal (supersymmetric or nonsupersymmetric) black holes is by now well understood [22, 23, 19]. Here we would like to present an interesting result on the existence of extremal black holes: we find that there are regular black hole solutions in a theory with only one gauge field (electric or magnetic) non-minimally coupled with the dilaton for non-trivial dilaton potentials. The attractor mechanism can be used then as a criterion for the existence of regular extremal black hole solutions. Similar considerations were made for baryonic and electromagnetic branes and other solutions in $A d S$ spacetime in [24]. More importantly, since the extremal (attractor) horizons are infinitely far away in the bulk 4 they do not get distorted by changing the boundary values of the scalars and so, from this point of view, the attractor mechanism acts as a no-hair theorem [1] for extremal black holes [13].

To understand why is so, let us review some basic facts related to the effective potential [26]. Since the dilaton is non-minimally coupled with the gauge field, a new term appears in its equation of motion. This term, which is controlled by the gauge field, can be interpreted as an effective potential for the dilaton. For example, for the spherically symmetric ansatz (38), an electromagnetic field with both electric and magnetic charges 5 turned on and the exponential coupling $e^{\phi}$, the effective potential is

$$
V_{e f f}(\phi)=\frac{1}{b^{2}}\left[e^{-\phi} Q^{2}+e^{\phi} P^{2}\right]+2 b^{2} V(\phi)
$$

Let us first discuss the case when the dilaton potential is vanishing $(V(\phi)=0)$. We observe that when just one charge is non-zero, the effective potential does not have a minimum at the horizon, which means that that there are no regular solutions in this case. When a second charge is turned on, the effective potential has a minimum and regular solutions exist. When the dilaton potential is non-zero, since there is a competition between the part generated by the gauge fields and pure dilaton part, the situation can drastically change. That is, there exist regular solutions even when just one charge is non-zero.

In principle, even if technically difficult, one could try to find some general conditions on the moduli potential so that the theory contains regular extremal solutions. However, such an analysis is beyond the goal of this paper and we prefer to prove the existence of this kind of solutions using the entropy function formalism of Sen [27] (see, also, [28] for stationary black holes). The important point is that the effective potential method and the entropy function formalism are equivalent in the near horizon limit 6 Since the near horizon geometry of extremal black holes has an enhanced symmetry of $A d S_{2} \times S^{2}$ (in the extremal case, the near horizon geometry is a solution of the equations of motion), the computations simplify drastically in the near horizon limit.

In what follows, we discuss the simplest potential (23), but the same kind of argument applies to more general potentials. The ansatz for the near horizon geometry of 4 -dimensional static extremal black holes is

$$
d s^{2}=v_{1}\left(-r^{2} d t^{2}+\frac{d r^{2}}{r^{2}}\right)+v_{2}\left[\frac{d y^{2}}{1-y^{2}}+\left(1-y^{2}\right) d \phi^{2}\right]
$$

By solving the attractor equations one can obtain the near horizon data, $\left\{v_{1}, v_{2}\right\}$, and also the horizon value of the dilaton $(u)$ as functions of the physical charge $q$ and the parameter $\alpha$ in the potential.

\footnotetext{
${ }^{4}$ It was shown in [25] that the near horizon geometry of extremal stationary black holes contains an $A d S_{2}$ spacetime.

${ }^{5}$ For two electric fields, to get regular solutions when the dilaton potential is turned off, the gauge couplings should be different so that the effective potential has a minimum at the horizon.

${ }^{6}$ Since we have exact solutions, we could find the near horizon metric directly for each solution. However such a computation is difficult and not very illuminating.
} 
After some straightforward computations we find

$$
\alpha=8 \frac{e^{u}}{q^{2}} \frac{2+u \sinh u-2 \cosh u}{[2(1+u)+u(\sinh u+\cosh u)-2 \cosh u-3 \sinh u]^{2}}
$$

We cannot solve this equation explicitly to obtain the horizon value of the dilaton. However for our purposes it is sufficient to observe that since the parameter $\alpha$ is arbitrary, we have the freedom to fix it so that the corresponding horizon value of the dilaton and the radii of $A d S_{2}$ and sphere are well defined at the horizon and so the solution is regular.

\section{Discussion}

In this paper we obtained asymptotically flat static solutions to the Einstein-Maxwell-dilaton system in the presence of a non-vanishing dilaton potential. Solving the field equations subject to a special ansatz for the metric and dilaton fields, we found regular solutions. Some of these contain examples that were considered previously in the literature and we have verified that our solutions (smoothly) reduce to these in the appropriate corners of parameter space. At this point, it is not clear to us how (or if it is possible) to embed our solutions into supergravity; this is an interesting open question that we leave for the future.

The no-hair theorem implies that short-range interactions decaying fast enough at infinity (hair) are not allowed in the exterior of a stationary black hole. Since the hair does not contribute to Gauss-like conserved charges, the classical degrees of freedom of the black hole are restricted to those related to its conserved charges. Over the past decade the accuracy of this theorem was strongly challenged. Indeed, hairy black hole solutions were discovered [5, 6, 7] that appear to evade the nohair theorems. Interestingly enough, we were able to obtain exact hairy asymptotically flat black hole solutions similar to the one predicted in [6]. To get a better understanding, let us now compare one of our dilaton potentials, the simplest one presented in (23), with a generic potential described in [6]. The main assumption in [6] is that the family of dilaton potentials used to construct the solution are not positive-semidefinite and the weak energy condition is not satisfied. Also, to obtain asymptotically flat solutions, the dilaton settles asymptotically at the local minimum, which should also correspond to a root of the potential. Therefore, the conditions imposed on the potentials of [6] for the existence of the asymptotically flat hairy black hole solutions are such that a root and a local minimum of the potential are located at the same place: $V\left(\phi_{\infty}\right)=\left.\partial_{\phi} V(\phi)\right|_{\phi_{\infty}}=0$ and $\left.\partial_{\phi \phi}^{2} V(\phi)\right|_{\phi_{\infty}}>0$. Now, let us consider the dilaton potential (23) and study its asymptotic behaviour for $\phi_{\infty}=0$. We obtain: $V(0)=\left.\partial_{\phi} V(\phi)\right|_{\phi=0}=\left.\partial_{\phi \phi}^{2} V(\phi)\right|_{\phi=0}=0$ and so the potential becomes very flat along this direction (in fact, the third and fourth derivatives vanish also at $\phi=0$ ). However, although the second derivative of the potential vanishes, the fifth does not and so the dilaton only feels the fifth derivative of the potential. We would like to point out that this potential resembles the potential of the Minimally Supersymmetric Standard Model (MSSM) flat direction inflation models (see, e.g., [29]) 7

When a classical scalar field acts as a source of gravity, the energy conditions can be violated depending on the form of the scalar potential and the value of the curvature coupling [30]. Let us consider again the simplest case (23). The sign of the potential is controlled by the sign of the parameter $\alpha$ - this can be easily seen by considering the derivatives in the Taylor expansion (close to the boundary) of the part of the potential that multiplies $\alpha$ and observing that all these derivatives are positive or zero. Therefore, a positive potential corresponds to a positive $\alpha$. However, we can explicitly check that, in this case and for $Q=P=0$, there are no black hole solutions, there exist

\footnotetext{
${ }^{7}$ The fields that compose flat directions may thus in principle be excited to large classical field strengths at no cost to the potential energy. Within MSSM, for example, all the flat directions are lifted by non-renormalizable operators.
} 
just naked singularities. When $\alpha$ is negative and $Q=P=0$ there exist regular black hole solutions but the weak energy condition is violated in agreement with the results of [30]. On the other hand, when $(Q, P)$ are non-zero, there exist regular black hole solutions even for a positive $\alpha$ and the weak energy condition is satisfied in this case.

The null energy condition is always satisfied. The energy momentum of the scalar field, in a comoving tetrad, has the form $T^{a b}=\operatorname{diag}\left(\rho, p_{1}, p_{2}, p_{2}\right)$ and satisfies the null energy condition. For our static solutions with the condition $f(x)>0$, we obtain

$$
\rho+p_{2}=0, \quad \rho+p_{1}=\frac{\left(\nu^{2}-1\right)\left(x^{\nu}-1\right)^{2} f(x)}{2 \nu^{2} x^{\nu+1}}>0 .
$$

For the non-extremal black holes, the near horizon geometry and the entropy vary continuously as the asymptotic values of moduli fields are changing. For example, we saw that for our solutions, the horizon radius depends of the horizon value of the dilaton which is controlled by the asymptotic data. On the other hand, for the extremal black holes, the moduli fields in a black hole background vary radially and get attracted to certain specific values at the horizon, which in fact depend only on the quantized charges of the black hole under consideration. It is then tempting to interpret that the attractor mechanism plays the role of a no-hair theorem for the extremal black holes [13]. As we have already described in detail in Section 4, due to the competition between the effective potential and dilaton potential, there exist also extremal black hole solutions with only one gauge field turned on.

We would like to comment now on the meaning of changing the dilaton's asymptotic value when the dilaton potential does not vanish. The equation (11) will have the same expression if we use the 'scaled' charges $\bar{Q}=e^{-\gamma \phi_{\infty}}$ and $\bar{P}=e^{\gamma \phi_{\infty}}$ and so the solutions have the same form if we replace the conserved charges with the 'scaled' charges. First, let us review what is happening when the dilaton potential vanishes [21]. Due to the non-minimal coupling of the dilaton, the first law should be supplemented by a new term containing the variation of the asymptotic value of the dilaton: $\Sigma d \phi_{\infty}$. Here, $\Sigma$ is the scalar charge that is defined as the monopole in the multipole expansion of the dilaton at infinity and $\phi_{\infty}$ is the asymptotic value of the dilaton. However, the correct interpretation for the existence of such a term was presented in [19]. That is, the new term does not have a similar interpretation like the other terms in the first law because it corresponds to a change in the couplings (so, by the variation of $\phi_{\infty}$ a different theory is obtained rather than another thermodynamic configuration). If one tries to use the scaling symmetry to get rid of this term, a different problem is encountered: the scaling symmetry does not preserve the conserved charges. The new solution cannot be reached dynamically starting from the old one because this will also force a violation of charge conservation. When the dilaton potential is not vanishing there is an even more drastic change. That is, by varying the asymptotic value of the dilaton, its potential is changing and, consequently, that effect corresponds to a change in the asymptotics of the solution.

Even if supersymmetry is experimentally discovered, one still has to understand how is it broken in the nature. It is possible to construct models for which the supersymmetry is broken spontaneously or by hand (as in our case). We have obtained solutions that are smoothly connected to some sugra solutions for which the microscopic entropy and the holographic (CFT) degrees of freedom are well understood. A related speculative observation is that it may be possible that our solutions could be described holographically by a dual QFT, which in some limit becomes a CFT and this is very similar to an RG flow towards a fixed point.

One important question is if these black holes are stable. Such an analysis is beyond the scope of this work. However, we would like to point out that in the extremal case the black holes are long lived states (metastable) that correspond to the minimum mass of the theory they belong to - the nonperturbative effects should play, in fact, an important role for understanding the stability of extremal 
black holes. This argument together with the attractor mechanism are at the basis of computing the microscopic entropy of extremal non-supersymmetric black holes in string theory [22, 19].

For future directions, one can study the phase structure of these black holes. This can be done, for example, by computing the quasilocal stress-tesor, action, and relevant thermodynamical quantities as in [31] Among other possible applications, one could find and describe the so-called 'hairosphere' [8]. We have explicitly shown that the non-linear character of the matter fields plays a key role in the construction of hairy black hole configurations. One interesting question is how close to a black hole event horizon can matter hover such that the non-linear behavior of the hair is present (in other words, how 'short' can hair be) [8] ?8 Since we have exact solutions, another possible application is to check the 'no-short-hair' conjecture of [8].

We would like to emphasize that with our method we can also generate solutions with scalars (and gauge fields) when the cosmological constant is positive - these solutions can be described along the lines of [32] (e.g., computing the corresponding c-functions). These solutions could provide interesting starting points for building novel cosmological scenarios.

\section{Acknowledgments.}

We would like to thank Andres Acena for important discussions and collaboration on related projects. DA would also like to thank David Choque for interesting conversations. Research of A.A. is supported in part by the Fondecyt Grant 11121187. The work of DA is supported by the Fondecyt Grant 1120446. This work was supported in part by the Natural Sciences and Engineering Research Council of Canada.

\section{A A general family of dilatonic dyonic black holes}

Let us consider the following action

$$
I\left[g_{\mu \nu}, A_{\mu}, \phi\right]=\frac{1}{2 \kappa} \int d^{4} x \sqrt{-g}\left[R-\frac{1}{4} e^{\lambda \phi} F^{2}-\frac{1}{2} \partial_{\mu} \phi \partial^{\mu} \phi-V(\phi)\right]
$$

The general class of solutions we present here is for $\lambda$ arbitrary and $\nu \rightarrow \infty$ and contains the solution presented in Section 2.1.1, which corresponds to $\lambda=1$. To obtain regular solutions in the limit $\nu \rightarrow \infty$ we should again rescale the coordinates as in Section 2.1.1 to obtain the following metric ansatz:

$$
d s^{2}=\Omega(x)\left[-f(x) d t^{2}+\frac{\eta^{2} d x^{2}}{x^{2} f(x)}+d \theta^{2}+\sin ^{2} \theta d \varphi^{2}\right]
$$

where again the conformal factor is

$$
\Omega(x)=\frac{x}{\eta^{2}(x-1)^{2}}
$$

With this form of the conformal factor, it is easy to obtain the dilaton and the $U(1)$ field strength:

$$
\phi=\ln (x) \quad, \quad A=\frac{Q}{x^{\lambda}} d t+P y d \varphi
$$

However, the dilaton potential and the metric are more complicated in this case. Let us first define the following expressions:

$$
\alpha_{1}=\eta^{2}\left(P^{2} \eta^{2}-\lambda^{2} Q^{2}\right), \quad \alpha_{2}=\eta^{2}\left(P^{2} \eta^{2}+\lambda^{2} Q^{2}\right),
$$

\footnotetext{
${ }^{8}$ In the asymptotic region, the behavior of the fields is dominated by linear terms in their respective equations of motion.
} 


$$
\begin{aligned}
W(h(\phi)) & =-\frac{\left(3 \lambda^{4}-9 \lambda^{2}+8\right) h(\lambda \phi)}{\left(\lambda^{2}-1\right) \lambda^{2}}-\frac{(\lambda+1) h((\lambda-2) \phi)}{2(\lambda-1)}-\frac{(\lambda-1) h((\lambda+2) \phi)}{2(\lambda+1)} \\
& +\frac{2\left(\lambda^{2}+\lambda-1\right)(\lambda-1) h((\lambda+1) \phi)}{(\lambda+1) \lambda^{2}}+\frac{2\left(\lambda^{2}-\lambda-1\right)(\lambda+1) h((\lambda-1) \phi)}{(\lambda-1) \lambda^{2}}
\end{aligned}
$$

where, for simplifying the notation, by $h(\phi)$ we indicate an arbitrary function of the dilaton. With these notations we can now write down the dilaton potential and the remaining unknown in the metric as

$$
\begin{gathered}
V(\phi)=\alpha_{1} W(\cosh (\phi))+\alpha_{2} W(\sinh (\phi))+4 \alpha_{0}(2 \phi+\phi \cosh (\phi)-3 \sinh (\phi)) \\
+\left(\frac{4 \alpha_{2}}{\lambda^{2}\left(\lambda^{2}-1\right)}\right)(\cosh (\phi)+2) \\
f_{\lambda}(x)=\frac{\eta^{2}(x-1)^{2} k}{x}+\left(x-\frac{1}{x}-2 \frac{\ln (x)}{x}\right) \alpha_{0}+\frac{\eta^{2}\left(\lambda(x-1)(x(\lambda+1)+1-\lambda)+2 x\left(1-x^{\lambda}\right)\right) Q^{2}}{\left(\lambda^{2}-1\right) x^{\lambda+1}} \\
+\frac{\eta^{4}\left(-2 x^{\lambda+1}\left(\lambda^{2}-1\right)+\lambda x^{\lambda}(\lambda+1)-2 x+\lambda x^{\lambda+2}(\lambda-1)\right) P^{2}}{\left(\lambda^{2}-1\right) x \lambda^{2}}
\end{gathered}
$$

\section{B A general family of electrically charged dilatonic black holes}

Let us consider again the action (63) and the dilaton expression as

$$
\phi=\sqrt{\nu^{2}-1} \ln (x), \quad l_{\nu}=\frac{1}{\sqrt{\nu^{2}-1}}
$$

A general class of solutions can be found when

$$
\lambda=\left(\frac{\nu+1}{\nu-1}\right)^{1 / 2}
$$

for which the metric and the gauge fields are

$$
d s^{2}=\Omega(x)\left(-f(x) d t^{2}+\frac{\eta^{2} d x^{2}}{f(x)}+d \Sigma_{k}\right) \quad, \quad A=-\frac{Q x^{-\nu}}{\nu} d t
$$

where the conformal factor and the other function in the metric are

$$
\Omega(x)=\frac{\nu^{2} x^{\nu-1}}{\eta^{2}\left(x^{\nu}-1\right)^{2}}
$$

Using $p=\lambda \sqrt{\nu^{2}-1}=\nu+1$ and $P=0$ in (14) we get

$$
\begin{aligned}
f(x) & =\frac{\eta^{2}}{\nu}\left(x^{2}+\frac{2 x^{2-\nu}}{\nu-2}-\frac{\nu}{\nu-2}\right)+f_{1}\left(\frac{x^{\nu+2}}{\nu+2}-x^{2}+\frac{x^{2-\nu}}{2-\nu}+\frac{\nu^{2}}{\nu^{2}-4}\right) \\
& -\frac{Q^{2} \eta^{2}}{\nu^{3}}\left(\frac{x^{2}}{2}+\frac{x^{2-2 \nu}}{2-2 \nu}-2 \frac{x^{2-\nu}}{2-\nu}-\frac{\nu^{2}}{(2-\nu)(2-2 \nu)}\right)
\end{aligned}
$$


Now, we set $f_{1}=\frac{\tilde{\alpha}}{\nu^{2}}-\frac{Q^{2} \eta^{2}(\nu+2)}{2 \nu^{3}(\nu-1)}+\frac{\eta^{2}(\nu+2)}{\nu^{2}}$ to get

$$
f(x)=\frac{\eta^{2} x^{2}\left(x^{\nu}-1\right)^{2}}{x^{\nu} \nu^{2}}+\frac{\tilde{\alpha}}{\nu^{2}}\left(\frac{x^{\nu+2}}{\nu+2}-x^{2}+\frac{x^{2-\nu}}{2-\nu}+\frac{\nu^{2}}{\nu^{2}-4}\right)-\frac{Q^{2} \eta^{2} x^{2-2 \nu}}{2 \nu^{3}(\nu-1)}\left(x^{\nu}-1\right)^{3}
$$

The dilaton potential is

$$
V(\phi)=\frac{2 \tilde{\alpha}}{\nu^{2}}\left(\frac{\nu-1}{\nu+2} \sinh \left((1+\nu) \phi l_{\nu}\right)+\frac{\nu+1}{\nu-2} \sinh \left((1-\nu) \phi l_{\nu}\right)+4 \frac{\nu^{2}-1}{\nu^{2}-4} \sinh \left(\phi l_{\nu}\right)\right) .
$$

In the limit $\nu=2$ we obtain the solution presented in Section 2.1.2, which is a smooth deformation of the electrically charged Kaluza-Klein black hole.

\section{References}

[1] W. Israel, "Event horizons in static vacuum space-times," Phys.Rev. 164 (1967) 1776-1779 B. Carter, "Axisymmetric Black Hole Has Only Two Degrees of Freedom," Phys.Rev.Lett. 26 (1971) 331-333

R. H. Price, "Nonspherical perturbations of relativistic gravitational collapse. 1. Scalar and gravitational perturbations," Phys.Rev. D5 (1972) 2419-2438

R. H. Price, "Nonspherical Perturbations of Relativistic Gravitational Collapse. II. Integer-Spin, Zero-Rest-Mass Fields," Phys.Rev. D5 (1972) 2439-2454

S. W. Hawking, "Breakdown of Predictability in Gravitational Collapse," Phys. Rev. D14 (1976) 2460-2473

J. D. Bekenstein, 'No Hair': Twenty-five Years After, chapter in Proceedings of the Second International Andrei D. Sakharov Conference in Physics, edited by I. M. Dremin and A. M. Semikhatov (World Scientific, Singapore, 1997).

[2] J. D. Bekenstein, "Novel 'no scalar hair' theorem for black holes," Phys. Rev. D 51, 6608 (1995).

[3] D. Sudarsky, "A Simple proof of a no hair theorem in Einstein Higgs theory," Class. Quant. Grav. 12, 579 (1995);

D. Sudarsky and T. Zannias, "Spherical black holes cannot support scalar hair," Phys. Rev. D 58 (1998) 087502 gr-qc/9712083.

[4] T. Hertog, "Towards a Novel no-hair Theorem for Black Holes," Phys. Rev. D 74, 084008 (2006) gr-qc/0608075.

[5] T. Torii, K. Maeda and M. Narita, "Scalar hair on the black hole in asymptotically anti-de Sitter space-time," Phys. Rev. D 64, 044007 (2001);

C. Martinez, R. Troncoso and J. Zanelli, "Exact black hole solution with a minimally coupled scalar field," Phys. Rev. D 70, 084035 (2004) hep-th/0406111;

E. Winstanley, "On the existence of conformally coupled scalar field hair for black holes in (antide Sitter space," Found. Phys. 33, 111 (2003) gr-qc/0205092;

A. Anabalon and J. Oliva, "Exact Hairy Black Holes and their Modification to the Universal Law of Gravitation," Phys. Rev. D 86, 107501 (2012) [arXiv:1205.6012 [gr-qc]];

J. Aparicio, D. Grumiller, E. Lopez, I. Papadimitriou and S. Stricker, "Bootstrapping gravity solutions," JHEP 1305, 128 (2013) [arXiv:1212.3609 [hep-th]];

W. Xu and L. Zhao, "Charged black hole with a scalar hair in (2+1) dimensions," arXiv:1305.5446 [gr-qc];

L. Zhao, W. Xu and B. Zhu, "Novel rotating hairy black hole in (2+1)-dimensions," 
arXiv:1305.6001 [gr-qc];

F. Correa, A. Faundez and C. Martinez, "Rotating hairy black hole and its microscopic entropy in three spacetime dimensions," Phys. Rev. D 87, 027502 (2013) arXiv:1211.4878 [hep-th]];

A. Anabalon, "Exact Hairy Black Holes," arXiv:1211.2765 [gr-qc];

A. Anabalon, F. Canfora, A. Giacomini and J. Oliva, "Black Holes with Primary Hair in gauged N=8 Supergravity," JHEP 1206 (2012) 010 arXiv:1203.6627 [hep-th]];

A. Anabalon and A. Cisterna, "Asymptotically (anti) de Sitter Black Holes and Wormholes with a Self Interacting Scalar Field in Four Dimensions," Phys. Rev. D 85 (2012) 084035 arXiv:1201.2008 [hep-th]];

A. Anabalon and H. Maeda, "New Charged Black Holes with Conformal Scalar Hair," Phys. Rev. D 81 (2010) 041501 arXiv:0907.0219 [hep-th]];

"Conformally coupled scalar black holes admit a flat horizon due to axionic charge," JHEP 1209 (2012) 008 arXiv:1205.4025 [hep-th]];

H. Lu, Y. Pang and C. N. Pope, "AdS Dyonic Black Hole and its Thermodynamics," arXiv:1307.6243 [hep-th].

[6] D. Sudarsky and J. A. Gonzalez, "On black hole scalar hair in asymptotically anti-de Sitter space-times," Phys. Rev. D 67, 024038 (2003) [gr-qc/0207069];

U. Nucamendi and M. Salgado, "Scalar hairy black holes and solitons in asymptotically flat space-times," Phys. Rev. D 68, 044026 (2003) gr-qc/0301062].

[7] A. Anabalon, "Exact Black Holes and Universality in the Backreaction of non-linear Sigma Models with a potential in (A)dS4," arXiv:1204.2720 [hep-th];

A. Acena, A. Anabalon and D. Astefanesei, "Exact hairy black brane solutions in $A d S_{5}$ and holographic RG flows," arXiv:1211.6126 [hep-th].

[8] D. Nunez, H. Quevedo and D. Sudarsky, "Black holes have no short hair," Phys. Rev. Lett. 76, 571 (1996) gr-qc/9601020.

[9] A. Anabalon and D. Astefanesei, "On attractor mechanism of $A d S_{4}$ black holes," arXiv:1309.5863 [hep-th].

[10] A. Salvio, "Holographic Superfluids and Superconductors in Dilaton-Gravity," JHEP 1209, 134 (2012) arXiv:1207.3800 [hep-th]];

A. Salvio, "Transitions in Dilaton Holography with Global or Local Symmetries," JHEP 1303, 136 (2013) arXiv:1302.4898 [hep-th]].

[11] G. W. Gibbons and K. -i. Maeda, "Black Holes and Membranes in Higher Dimensional Theories with Dilaton Fields," Nucl. Phys. B 298, 741 (1988).

[12] S. Ferrara, R. Kallosh, and A. Strominger, N=2 extremal black holes, Phys. Rev. D52 (1995) 5412-5416, [hep-th/9508072];

A. Strominger, Macroscopic entropy of $n=2$ extremal black holes, Phys. Lett. B383 (1996) 39-43, [hep-th/9602111];

S. Ferrara and R. Kallosh, Supersymmetry and attractors, Phys. Rev. D54 (1996) 1514-1524, [hep-th/9602136];

S. Ferrara and R. Kallosh, Universality of supersymmetric attractors, Phys. Rev. D54 (1996) 1525-1534, [hep-th/9603090].

[13] D. Astefanesei, H. Nastase, H. Yavartanoo and S. Yun, "Moduli flow and non-supersymmetric AdS attractors," JHEP 0804, 074 (2008) [arXiv:0711.0036 [hep-th]]; 
D. Astefanesei, N. Banerjee and S. Dutta, "(Un)attractor black holes in higher derivative AdS gravity," JHEP 0811, 070 (2008) [arXiv:0806.1334 [hep-th]].

[14] D. Garfinkle, G. T. Horowitz and A. Strominger, "Charged black holes in string theory," Phys. Rev. D 43 (1991) 3140 [Erratum-ibid. D 45 (1992) 3888].

[15] R. Gregory and J. A. Harvey, "Black holes with a massive dilaton," Phys. Rev. D 47 (1993) 2411 hep-th/9209070.

[16] C. Charmousis, B. Gouteraux and J. Soda, "Einstein-Maxwell-Dilaton theories with a Liouville potential," Phys. Rev. D 80 (2009) 024028 [arXiv:0905.3337 [gr-qc]].

[17] P. Dobiasch and D. Maison, "Stationary, Spherically Symmetric Solutions Of Jordan's Unified Theory Of Gravity And Electromagnetism," Gen. Rel. Grav. 14, 231 (1982).

[18] G. W. Gibbons and D. L. Wiltshire, "Black Holes in Kaluza-Klein Theory," Annals Phys. 167, 201 (1986) [Erratum-ibid. 176, 393 (1987)].

[19] D. Astefanesei, K. Goldstein and S. Mahapatra, "Moduli and (un)attractor black hole thermodynamics," Gen. Rel. Grav. 40, 2069 (2008) hep-th/0611140].

[20] R. Kallosh, A. D. Linde, T. Ortin, A. W. Peet and A. Van Proeyen, "Supersymmetry as a cosmic censor," Phys. Rev. D 46, 5278 (1992) hep-th/9205027).

[21] G. W. Gibbons, R. Kallosh and B. Kol, "Moduli, scalar charges, and the first law of black hole thermodynamics," Phys. Rev. Lett. 77, 4992 (1996) [hep-th/9607108].

[22] A. Dabholkar, A. Sen and S. P. Trivedi, "Black hole microstates and attractor without supersymmetry," JHEP 0701, 096 (2007) hep-th/0611143.

[23] A. Sen, "Black Hole Entropy Function, Attractors and Precision Counting of Microstates," Gen. Rel. Grav. 40, 2249 (2008) [arXiv:0708.1270 [hep-th]].

[24] D. Astefanesei, N. Banerjee and S. Dutta, "Moduli and electromagnetic black brane holography," JHEP 1102, 021 (2011) [arXiv:1008.3852 [hep-th]];

D. Astefanesei, N. Banerjee and S. Dutta, "Near horizon data and physical charges of extremal AdS black holes," Nucl. Phys. B 853, 63 (2011) arXiv:1104.4121 [hep-th]].

[25] H. K. Kunduri, J. Lucietti and H. S. Reall, "Near-horizon symmetries of extremal black holes," Class. Quant. Grav. 24, 4169 (2007) arXiv:0705.4214 [hep-th]];

D. Astefanesei and H. Yavartanoo, "Stationary black holes and attractor mechanism," Nucl. Phys. B 794, 13 (2008) arXiv:0706.1847 [hep-th]].

[26] K. Goldstein, N. Iizuka, R. P. Jena, and S. P. Trivedi, Non-supersymmetric attractors, Phys. Rev. D72 (2005) 124021, [hep-th/0507096].

[27] A. Sen, Black hole entropy function and the attractor mechanism in higher derivative gravity, JHEP 09 (2005) 038, [hep-th/0506177];

A. Sen, Entropy function for heterotic black holes, JHEP 03 (2006) 008, [hep-th/0508042].

[28] D. Astefanesei, K. Goldstein, R. P. Jena, A. Sen, and S. P. Trivedi, Rotating attractors, JHEP 10 (2006) 058, [hep-th/0606244]. 
[29] R. Allahverdi, K. Enqvist, J. Garcia-Bellido, A. Jokinen and A. Mazumdar, "MSSM flat direction inflation: Slow roll, stability, fine tunning and reheating," JCAP 0706, 019 (2007) hep-ph/0610134.

[30] E. E. Flanagan and R. M. Wald, "Does back reaction enforce the averaged null energy condition in semiclassical gravity?," Phys. Rev. D 54, 6233 (1996) gr-qc/9602052;

C. Barcelo and M. Visser, "Scalar fields, energy conditions, and traversable wormholes," Class. Quant. Grav. 17, 3843 (2000) gr-qc/0003025;

M. Salgado, D. Sudarsky and U. Nucamendi, "The Violation of the weak energy condition, is it generic of spontaneous scalarization?," Phys. Rev. D 70, 084027 (2004) gr-qc/0402126.

[31] D. Astefanesei and E. Radu, "Quasilocal formalism and black ring thermodynamics," Phys. Rev. D 73, 044014 (2006) hep-th/0509144;

R. B. Mann and D. Marolf, "Holographic renormalization of asymptotically flat spacetimes," Class. Quant. Grav. 23, 2927 (2006) hep-th/0511096];

D. Astefanesei, R. B. Mann, M. J. Rodriguez and C. Stelea, "Quasilocal formalism and thermodynamics of asymptotically flat black objects," Class. Quant. Grav. 27, 165004 (2010) arXiv:0909.3852 [hep-th]];

D. Astefanesei, M. J. Rodriguez and S. Theisen, "Thermodynamic instability of doubly spinning black objects," JHEP 1008, 046 (2010) [arXiv:1003.2421 [hep-th]].

[32] F. Leblond, D. Marolf and R. C. Myers, "Tall tales from de Sitter space 1: Renormalization group flows," JHEP 0206, 052 (2002) hep-th/0202094;

D. Astefanesei, R. B. Mann and E. Radu, "Reissner-Nordstrom-de Sitter black hole, planar coordinates and dS / CFT," JHEP 0401, 029 (2004) hep-th/0310273. 